\title{
Adenomatoid odontogenic tumour displacing multiple teeth in an adolescent
}

\author{
Kellen Cristine Tjioe ${ }^{*}$, Denise Tostes Oliveira, Marcelo Lupion Poleti, Osny Ferreira Jr., \\ Eduardo Sant'Anna, Eduardo Sanches Gonçales \\ Department of Stomatology, Bauru School of Dentistry, University of São Paulo, Bauru, Brazil \\ Email: "kellentjioe@yahoo.com.br, eduardogoncales@usp.br
}

Received 24 February 2012; revised 18 March 2012; accepted 28 April 2012

\begin{abstract}
Adenomatoid odontogenic tumor is an unusual benign neoplasm which shares clinical and radiographic characteristics with odontogenic cystic lesions denoting a distinct behavior. This paper reports a case of a 12-year-old boy with a bony consistence tumefaction involving the area from teeth 12 to 17 . Radiographically, the lesion was characterized by a well-circumscribed unilocular radiolucent area displacing maxillary canine, first and second premolars. The final diagnosis was adenomatoid odontogenic tumour. The lesion was enucleated concomitantly with the removal of the canine tooth under general anesthesia. The patient was submitted to orthodontic treatment and has been followed-up for four years. Clinical and radiographic features are discussed in this report.
\end{abstract}

Keywords: Odontogenic Tumor; Adenomatoid Odontogenic Tumor; Oral Surgery

\section{INTRODUCTION}

The odontogenic tumors comprise a group of rare neoplasias that corresponds to about $1 \%$ of all tumors [1]. It is believed that they are derived from the remnant tissue of tooth formation. The adenomatoid odontogenic tumor (AOT), on the other hand, accounts for $2.2 \%-7.1 \%$ of odontogenic tumors [2-4]. It is a benign neoplasm which affects predominantly the jaws of female adolescents [1, 2,5-7]. The typical appearance of an AOT is a well-defined radiolucent lesion containing radiopaque foci in its interior. Frequently, it is surrounding a tooth [1,2,5-7].

Here we report an unusual case of an AOT affecting a male adolescent with distinct epidemiological, clinical and radiographic features.

\section{CASE REPORT}

A 12-year-old boy was referred to our service for the

\footnotetext{
"Corresponding author.
}

assessment of a swelling in the gingiva with unknown time of evolution. Local inspection revealed a tumefaction involving the area from teeth 12 to 17 , with bony consistence and healthy overlying mucosa. Dental examination discarded infectious origin of the lesion. Panoramic radiograph revealed a well-circumscribed unilocular radiolucent area extending from tooth 12 to 17, measuring approximately $3.5 \times 4 \mathrm{~cm}$ in diameter (Figure 1). The canine, first and second premolar teeth were displaced by the lesion and the canine was partially embedded by it. No radiopacity within the lesion could be seen as well as root resorption of any permanent tooth. The floor of the maxillary sinus was highly displaced, limiting superiorly the lesion (Figure 2).

The aspirative biopsy was performed and showed the collection of a translucent yellowish fluid. In sequence, the incisional biopsy was performed. Microscopical evaluation of the material revealed a virtual cystic cavity (Figure 3). In the well-defined wall, composed by cuboidal epithelium, it was possible to observe cords and strands of epithelium interlaced with duct-like structures in a scant fibrous stroma. Moreover, amorphous basophilic material was found. Based on these findings the final diagnosis was adenomatoid odontogenic tumor.

The lesion was enucleated concomitantly with the removal of the canine tooth under general anesthesia (Figure 4). The patient has been followed-up for four years and was submitted to orthodontic treatment. No recurrence was found (Figure 5).

\section{DISCUSSION}

The adenomatoid odontogenic tumor is a benign neoplasm which affects the jaw bones. There are two subtypes of AOT: follicular and extrafollicular. The first one is the most common type and is characterized by a well-defined unilocular radiolucency surrounding the crown and is often part of the root of an unerupted tooth, usually the canine tooth. This association may prevent the eruption of that tooth [8].

AOT is a tumor of young people. About one third of 


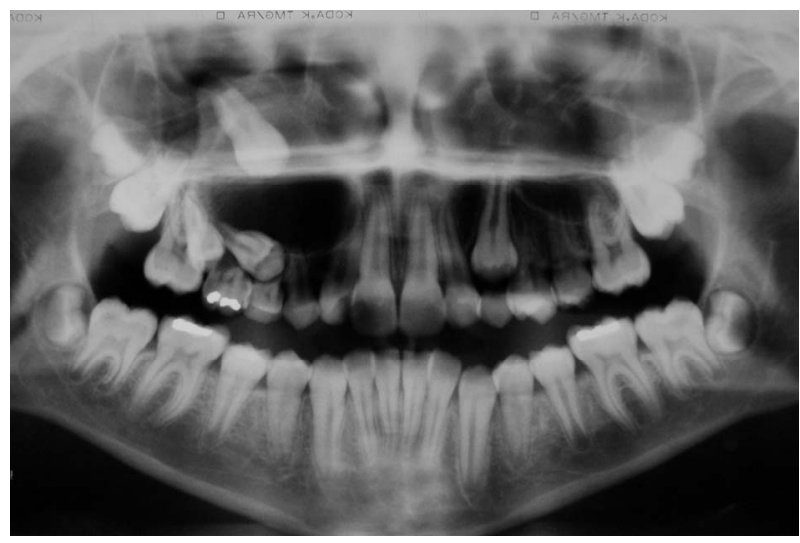

Figure 1. Panoramic radiograph showing a well-circumscribed unilocular radiolucent area extending from tooth 12 to 17, displacing the canine and premolar teeth.

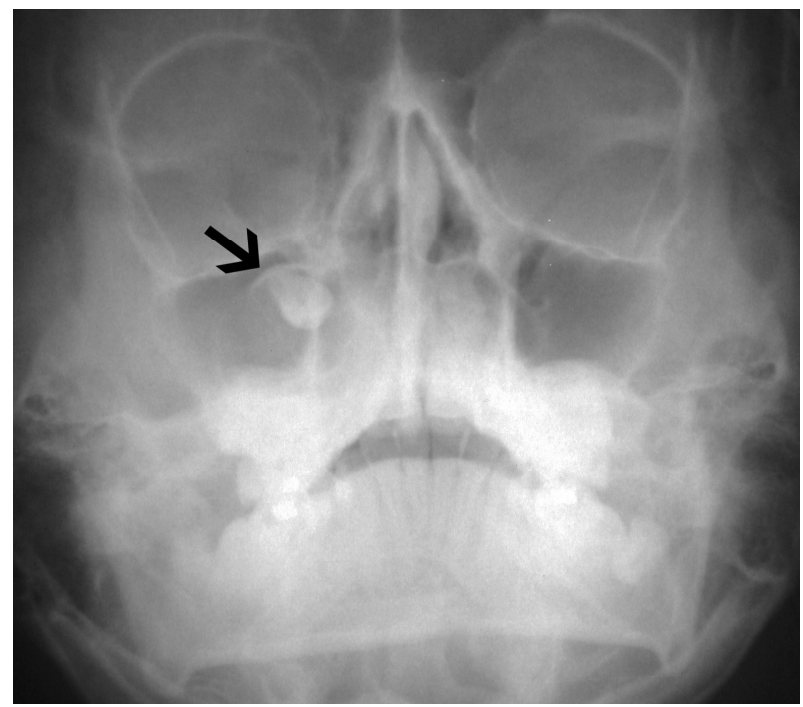

Figure 2. Water's projection highlighting the superior limit of the lesion (black arrow).

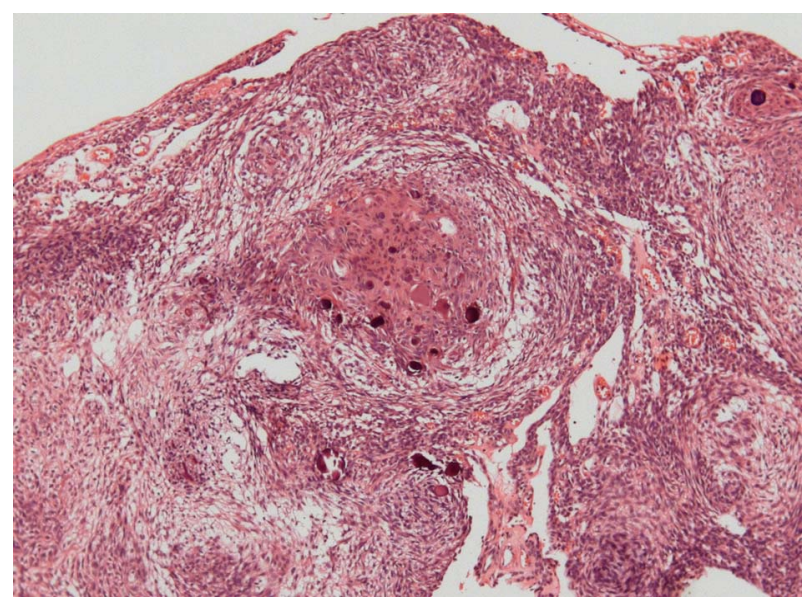

Figure 3. Histopathologic features of the lesion showing the characteristic spindle-shaped cells interlaced with duct-like structures. Amorphous basophilic material can be seen too (Hematoxylin-eosin staining, original magnification $\times 100$ ).

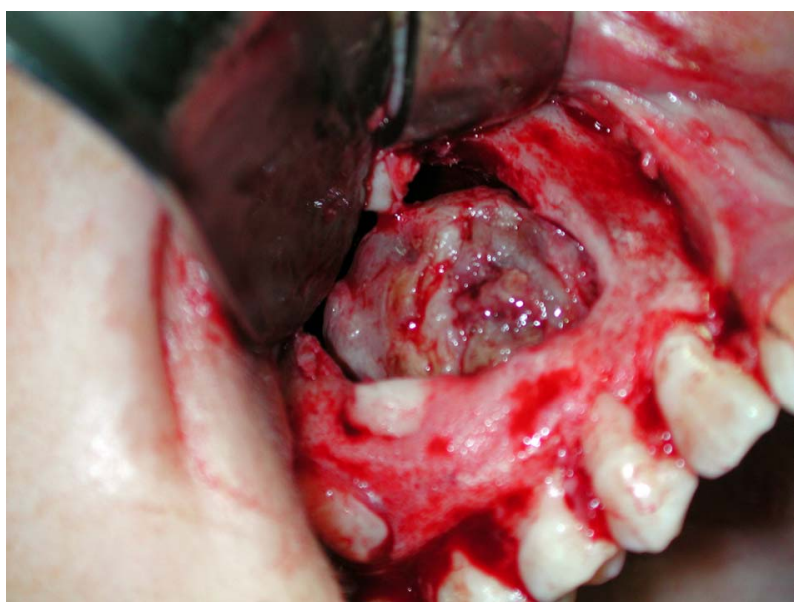

Figure 4. Trans-operatory view. The well-encapsulated lesion was successfully removed from the cavity.

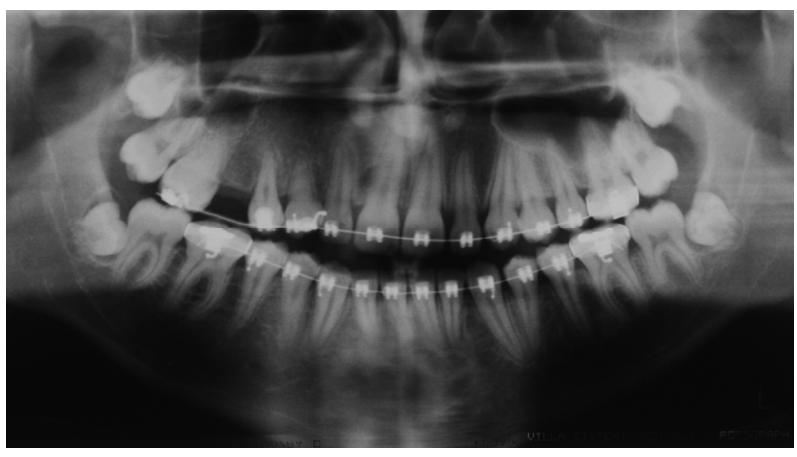

Figure 5. Four-year-follow-up radiograph after the surgery.

them takes place between 10 and 19 years and $88 \%$ of AOT are diagnosed during the second and third decades of life [3], as occurred in the presented case. On the other hand, we report here a case of AOT in a boy while women are approximately twice more affected than men in the first decades [2,7].

AOT is unique among the other odontogenic tumors, due to the very limited period of occurrence and a quite restricted anatomic location (80\% in the incisor-canine region [2,7,8] for its onset. Most cases are between 1 and $3 \mathrm{~cm}$ in greatest diameter and $71 \%$ are associated with an impacted tooth [3]. This report shows a huge lesion involving multiple teeth. The total displacement of the maxillary canine tooth to the superior boundary of the lesion and the presence of the radiopaque halo near to the amelo-cementary junction led us to conjecture that the neoplastic mass may be connected to this tooth, what was verified during the surgical procedure. Its progressive slow growth dislocated the adjacent teeth to the lateral and inferior aspects of the tumor while the associated tooth was putted in intimate contact with the maxillary sinus, which walls were severally misshapen. Indeed, the Water's projection was useful to evaluate the height of the canine. Still, it was not possible to see the 
typical radiopaque foci within the radiolucent lesion, found in $65 \%$ of cases of AOT. Probably because the panoramic technique is not the one of choice to evaluate details of the periapical area. Still, the incisional biopsy was mandatory in the case to reach the precise diagnosis.

Despite of the significant proportion the tumor took, it is well established the benign course and good prognosis of this neoplasia. Therefore, the conservative complete surgical excision of the lesion is the treatment of choice $[2,3,6]$. The recurrence is rare $[2,3,6]$.

This case report contributes to reinforce that careful diagnostic procedures and adequate interpretation of radiographic findings are indispensable to reach a pertinent diagnosis, in order to make the correct treatment of the disease.

\section{REFERENCES}

[1] Ochsenius, G., Escobar, E., Godoy, L. and Penafiel, C. (2007) Odontogenic cysts: Analysis of 2944 cases in Chile. Medicina Oral, Patologia Oral y Cirugia Bucal, 2, 85-91.

[2] Philipsen, H.P. and Reichart, P.A. (1999) Adenomatoid odontogenic tumour: Facts and figures. Oral Oncology, 35, 125-131. doi:10.1016/S1368-8375(98)00111-0

[3] Rick, G.M. (2004) Adenomatoid odontogenic tumor. Oral and Maxillofacial Surgery Clinics of North America, 16, 333-354. doi:10.1016/j.coms.2004.04.001

[4] Swasdison, S., Dhanuthai, K., Jainkittivong, A. and Philipsen, H.P. (2008) Adenomatoid odontogenic tumors: An analysis of 67 cases in a Thai population. Oral Surgery, Oral Medicine, Oral Pathology, Oral Radiology, and Endodontics, 105, 210-215. doi:10.1016/j.tripleo.2007.02.020

[5] Fernandes, A.M., Duarte, E.C., Pimenta, F.J., Souza, L.N., Santos, V.R., Mesquita, R.A., et al. (2005) Odontogenic tumors: A study of 340 cases in a Brazilian population. Journal of Oral Pathology \& Medicine, 34, 583-587. doi:10.1111/j.1600-0714.2005.00357.x

[6] Philipsen, H.P. and Nikai, H. (2005) The adenomatoid odontogenic tumour. In: Barnes, L., Eveson, J.W., Reichart, P. and Sidransky, D., Eds., Pathology and Genetics of Head and Neck Tumours, IARC Press, Lion, 304-305.

[7] Philipsen, H.P., Reichart, P.A., Siar, C.H., Ng, K.H., Lau, S.H., Zhang, X., et al. (2007) An updated clinical and epidemiological profile of the adenomatoid odontogenic tumour: A collaborative retrospective study. Journal of Oral Pathology \& Medicine, 36, 383-393. doi:10.1111/j.1600-0714.2007.00536.x

[8] Ide, F., Mishima, K., Kikuchi, K., Horie, N., Yamachika, S., Satomura, K., et al. (2011) Development and growth of adenomatoid odontogenic tumor related to formation and eruption of teeth. Head Neck Pathol, 5, 123-132. doi:10.1007/s12105-011-0253-3 